\title{
REVISIÓN DE ALGORITMOS DE VERIFICACIÓN AUTOMÁTICA \\ DE FIRMAS OFF-LINE
}

\author{
Luis Enrique Vílchez Fernández* \\ evilchezfernandez@gmail.com \\ Oficina Nacional de Procesos Electorales - ONPE, Lima, Perú
}

Resumen

En la actualidad, la firma es uno de los distintivos más aceptados para la identificación personal. Su inclusión es obligatoria en documentos como cheques bancarios, contratos, tarjetas de crédito, entre otros documentos públicos y privados. No obstante, la firma se ha convertido en un atractivo objetivo para las falsificaciones y, en consecuencia, para el fraude. Por esta razón, se han realizado investigaciones en soluciones automatizadas de reconocimiento de firmas y estudios del estado del arte que ahora resulta necesario actualizar, puesto que el trabajo más exhaustivo data del 2008 y en los años siguientes se han efectuado nuevas investigaciones. El presente trabajo se enfoca en el estudio comparativo de las técnicas de verificación de firmas off-line desde los puntos de vista de eficiencia y exactitud para verificar la autenticidad de la persona. La metodología de investigación utilizada considera el procedimiento propuesto por Kitchenham, el cual ha sido adaptado e involucra las fases de planeamiento, desarrollo y reportes de la revisión.

Palabras clave: Verificación de firmas / reconocimiento de firmas / firmas off-line

Abstract

Nowadays, the signature is one of the most accepted badges for personal identification. Its inclusion is mandatory in documents such as bank checks, contracts, credit cards, among other public and private documents. However, the signature has become an attractive target for counterfeiting and, consequently, for fraud. For this reason, research has been carried out on automated signature recognition and state-of-the-art studies that are now required to be updated, since the most comprehensive work dates back to 2008 and in the following years further research has been carried out. The present work focuses on the comparative study of verification techniques of signatures off-line from the point of view of efficiency and accuracy to verify the person's authenticity. The research methodology used considers the procedure proposed by Kitchenham, which has been adapted, and involves the phases of planning, development and reporting of the review.

Keywords: Verification of signatures / recognition of signatures / signatures off-line 


\section{Introducción}

En la actualidad, la firma es considerada como uno de los atributos más aceptados para la identificación personal, a tal punto que su inclusión es obligatoria en documentos como cheques bancarios, contratos, tarjetas de crédito, documentos públicos y privados, etcétera (Batista, Granger y Sabourin, 2010).

El efecto secundario del uso de las firmas radica en que estas pueden ser explotadas con el fin de simular la autenticidad de un documento y ha sido un objetivo atractivo para el fraude desde hace mucho tiempo (Vargas, Ferrer, Travieso y Alonso, 2011). Por ejemplo, en el Perú, para la inscripción de organizaciones políticas locales y regionales, el Registro Nacional de Identificación y Estado Civil (Reniec) ha recibido 8392714 firmas, de las cuales 3750389 fueron registros inválidos.

Las falsificaciones de firmas generalmente se dividen en tres subconjuntos diferentes: aleatorias, simples y simuladas. La falsificación aleatoria es generalmente una muestra genuina de la firma que pertenece a un escritor diferente; la falsificación simple se produce cuando el falsificador conoce el nombre del escritor pero no tiene acceso a una muestra de la firma; y la falsificación simulada es una imitación razonable del modelo de la firma genuina (Bertolini, Oliveira, Justino y Sabourin, 2010). Esta última es la más difícil de detectar porque la imitación puede parecer real a simple vista, pero siempre deja algún detalle fuera del patrón de la genuina.

Debido a este problema, se han llevado a cabo investigaciones en soluciones eficientes automatizadas de reconocimiento de firmas, que se dividen en dos categorías: verificación on-line y verificación off-line. La verificación de firmas on-line es aquella en la cual la firma es capturada con dispositivos especiales (lápiz electrónico y una tableta) y permite obtener características dinámicas de la firma, como son: velocidad de escritura, aceleración, presión del lápiz electrónico, orden de los trazos, ángulo formado entre el lápiz electrónico y una tableta. Por su parte, la verificación de firmas off-line es aquella que usa un escáner óptico para obtener los datos de las firmas registradas en un papel, por lo que es necesario aplicar técnicas de procesamiento de imágenes complejas para los segmentos de la firma y analizar su forma para la extracción de características (Vargas et al., 2011). Esta última categoría es la más común, puesto que no requiere de dispositivos de procesamiento especiales cuando se produce la firma y, en consecuencia, su aplicación es mucho más práctica.

En los últimos años se han desarrollado diversos algoritmos para realizar la verificación automática de firmas offline, como redes neuronales (Kumar, Sharma y Chanda, 2012), máquina de vectores de soporte (Vargas et al., 2011), modelos ocultos de Markov (Wen, Fang, Tang y Zhang, 2009), métodos difusos (Kudłacik y Porwik, 2014), métodos basados en la distancia (Rico e Iñesta, 2012), entre otros. Asimismo, se han llevado a cabo trabajos 
del estado del arte que estudian y documentan las investigaciones efectuadas en el campo de la verificación automática de firmas (Plamondon y Lorette, 1989), (Leclerc y Plamondon, 1994), (Impedovo y Pirlo, 2008), de los cuales el trabajo más exhaustivo fue el presentado en el 2008. Sin embargo, se han elaborado nuevos algoritmos, por lo que se hace necesario actualizar una revisión de algoritmos, una clasificación y una evaluación comparativa.

El presente artículo se enfoca en el estudio comparativo de las técnicas de verificación de firmas offline desde los puntos de vista de eficiencia y exactitud, y está organizado en cuatro secciones. En la siguiente sección se describe la metodología de investigación seguida para este estudio. El análisis de la investigación de los artículos y los resultados estadísticos son mostrados en la sección 3. Finalmente, las conclusiones son presentadas en la sección 4.

\section{Metodología de la investigación}

En el levantamiento de información para este trabajo, se realizó el procedimiento propuesto por Kitchenham et al. (2009) en las fases de planificación, conducción y reportes de la revisión. En la planificación de la revisión se formulan cinco preguntas, que contribuirán a esclarecer los objetivos de la investigación.

Q1: ¿Qué trabajos se han desarrollado para la adquisición y preprocesamiento de firmas?

Q2: ¿Qué características se han identificado para verificar firmas?

Q3: ¿Qué algoritmos se han desarrollado para la verificación (clasificación)?

Q4: ¿Qué datasets se han utilizado para validar los métodos de verificación de firmas?

Q5: ¿Cuál es el rendimiento que presentan los trabajos de verificación de firmas?

Las fuentes de información consultadas para responder las preguntas planteadas fueron Ios siguientes bancos: IEEE Xplore, ACM Digital Library, Springer, Science Direct, DOAJ, Taylor and Francis, y Emerald. La investigación cubre el periodo comprendido entre el 2008 y el 2016, considerando que el trabajo del estado arte de Impedovo y Pirlo (2008) es uno de los trabajos más exhaustivos sobre verificación automática de firmas. Las claves usadas para la búsqueda de fuentes en esos bancos se obtuvieron con la combinación de las palabras verification of signatures, recognition of signatures, signatures offline, handwriting recognition y fueron aplicadas al título, al abstract y a las keywords.

Los criterios usados para la selección y exclusión de fuentes, incluidos journals con factor de impacto SJR, son indicados en la tabla 1. 
Tabla 1. Criterios considerados para inclusión y exclusión de fuentes de referencia

\section{Selección}

Exclusión

- Presentan modelos, algoritmos, herramientas para la verificación de firmas offline

- Trabajos de verificación de firmas que no aportan a su automatización o a la extracción de atributos

- Proponen caracterización o formas de obtenerlos

- Trabajos dedicados solo a reconocimiento de firmas online

- Responden directamente a las preguntas de la investigación

- Trabajos de revisión de literaturas genéricas

- Proponen métricas para la medición de la precisión de verificación de firmas

- Pósteres, editoriales, libros y tesis

Elaboración propia

En la conducción de la revisión, las informaciones potenciales identificadas en el proceso de búsqueda, con un resultado de 11577 fuentes, fueron sometidas a un proceso de selección de acuerdo a los criterios de inclusión y exclusión establecidos en la tabla 1, previamente a una revisión de contenidos, a fin de determinar su relevancia para el presente estudio, seleccionándose 37 trabajos de investigaciones relacionados con la verificación automática de firmas (ver tabla 2).

El proceso aplicado, así como los resultados obtenidos en cada paso del proceso, se representan en la figura 1.

Figura 1. Proceso aplicado para la revisión sistemática de literatura

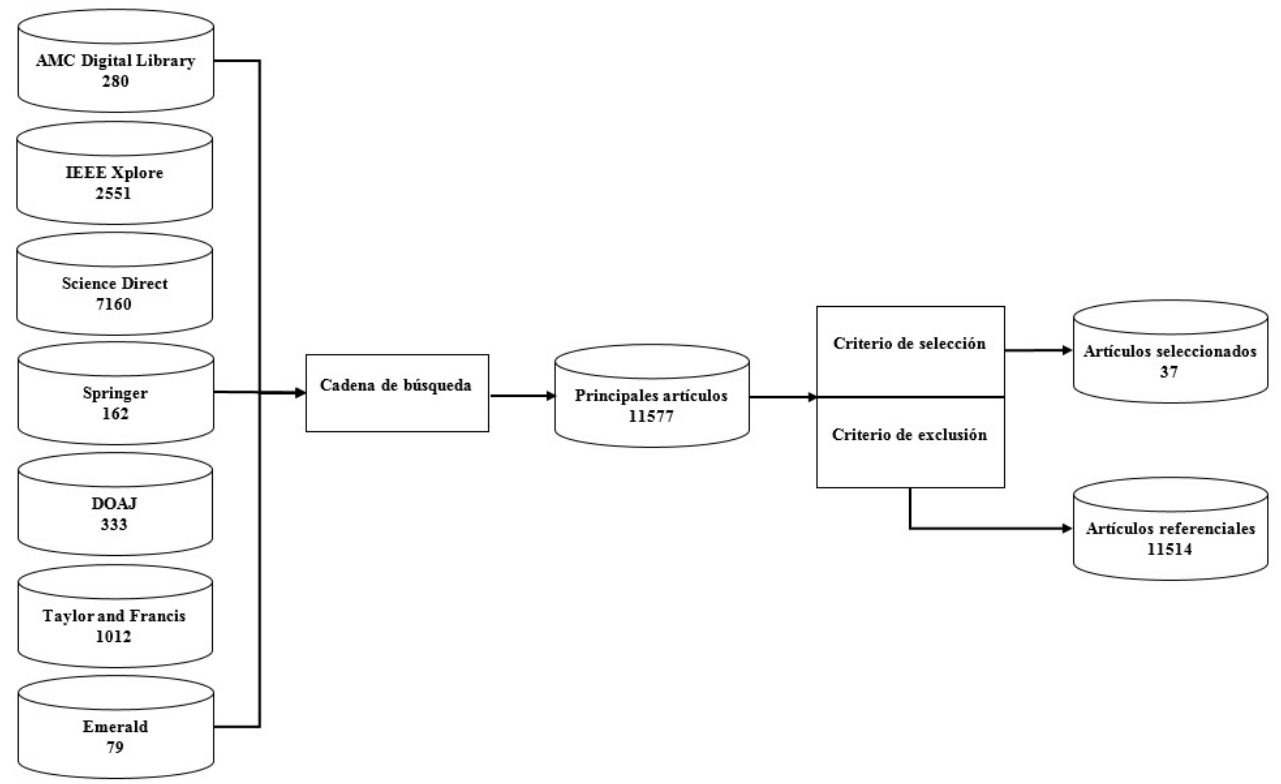


Tabla 2. Estudios potencialmente elegibles y estudios seleccionados

\begin{tabular}{|l|c|c|}
\multicolumn{1}{|c|}{ Fuente } & Estudios potencialmente elegibles & Estudios seleccionados \\
\hline ACM Digital Library & 280 & 1 \\
IEEE Xplore & 2551 & 9 \\
Science Direcct & 7160 & 11 \\
Springer & 162 & 9 \\
DOAJ & 333 & 5 \\
Taylor and Francis & 1012 & 1 \\
Esmerald & 79 & 1 \\
\hline Total & 11577 & 37 \\
\hline
\end{tabular}

Elaboración propia

El estudio y análisis de las fuentes seleccionadas a fin de responder las preguntas de la investigación, se abordan en la siguiente sección.

\section{Análisis y reportes}

Impedovo y Pirlo (2008) consideran tres fases principales en el proceso de verificación automática de firmas: adquisición de datos y preprocesamiento, extracción de características y clasificación (figura 2).

Figura 2. Proceso de verificación automática de firmas

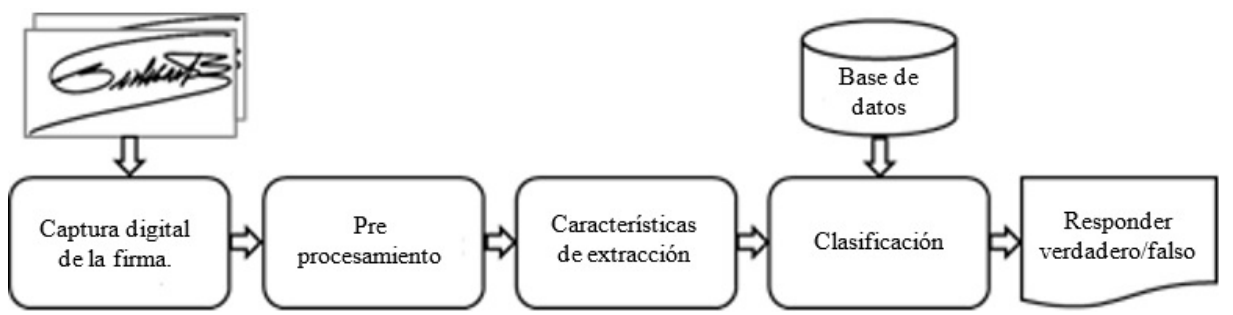

Elaboración propia

En esencia, los estudios seleccionados se enfocan en las fases de extracción de atributos y las clasificaciones, con algunas variaciones en el preprocesamiento; tópicos que serán analizados en detalle en las siguientes subsecciones, a fin de responder a nuestras preguntas de investigación. 


\subsection{Q1: ¿Qué trabajos se han desarrollado para la adquisición y preprocesamiento de firmas?}

La adquisición de datos para la verificación de firmas offline se realiza después de que la firma se ha completado y plasmado en un documento, a través de un escáner óptico (Batista et al., 2010). En el preprocesamiento la imagen es preparada para realizar una eficiente extracción de características. Generalmente la imagen es binarizada por Tsu, pero algunos enfoques operan en tonalidad de gris, tal como señalan Vargas et al. Los ruidos de la imagen son eliminados mediante el uso de operaciones de filtro morfológico (Shekar, Bharathi, Kittler, Vizilter y Mestestskiy, 2015).

Las firmas pueden ser registradas con bolígrafos de tintas de alguna tonalidad de color en formato RGB, las cuales generalmente son convertidas a escala de gris y posteriormente en binarios, como lo descrito por Radhika, Venkatesha y Sekhar (2011). Ferrer, Vargas, Morales y Ordóñez (2012) remueven el fondo de gris y binarizan por posterización; estandarizan el espesor del trazo de la firma con operaciones topológicas, remoción de puntos aislados y de fondo. Wen et al. (2009), adicionalmente, esqueletizan el trazo de la firma a un pixel. Tselios, Zois, Siores, Nassiopoulos y Economou (2012) adelgazan la firma a trazos de 1 pixel de ancho, se corta la imagen a un 80 \% de su ancho y largo en el centro de gravedad, con el propósito de eliminar trazos externos y quedar con el área con más información de la firma.

El ruido generado en el proceso de adquisición es reducido con la aplicación de técnicas como filtro de la mediana (Pirlo e Impedovo, 2013b; Radhika et al., 2011). Kumar et al. (2012) aplican en la imagen binaria el análisis de componentes conectados, definiendo un umbral apropiado de tal forma que las partículas aisladas de tamaño menor que el umbral sean removidas. En algunos casos, para mantener buena conectividad de los trazos de la firma, los niveles de intensidades son intercambiados con la operación de XOR, se adelgaza la firma y después se invierte la operación para obtener una firma de líneas suavemente conectadas (Radhika et al., 2011).

Jena, Majhi, Panigrahy y Jena (2008) ajustan la imagen de ROI al menor tamaño con el método bounding box mínimo, orientado en función de ejes principales que posibilitan la corrección de inclinación. Wen et al. (2009) estiman que la corrección de inclinación altera algunos detalles naturales de las firmas y prefieren usar métodos invariantes en extracción de atributos. La figura 3 muestra el resultado de cada una de las etapas del preprocesamiento. 
Figura 3. (a) Imagen en escala de grises, (b) Imagen binarizada, (c) Ruido eliminado, (d) Esqueletización y rotación de la imagen, (e) Imagen normalizada

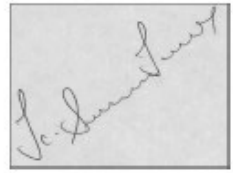

(a)

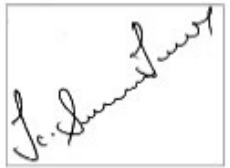

(b)

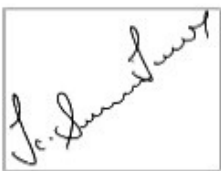

(c)

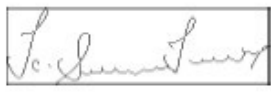

(d)

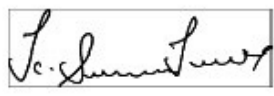

(e)

Fuente: Kumar et al. (2012)

\subsection{Q2: ¿Qué características se han identificado para verificar firmas?}

La selección y extracción de características de las firmas off-line es la parte más importante en el proceso de verificación; estas características se clasifican en dos principales categorías: parámetros globales y parámetros locales. Los parámetros globales se refieren a toda la firma; mientras que los parámetros locales, dependiendo del nivel de detalle considerado pueden dividirse en dos grupos: orientados a componentes (por ejemplo, la relación de altura y anchura de los trazos, posición relativa de los trazos, orientación de los trazos, etcétera) y orientados a pixel (por ejemplo, la densidad del pixel, intensidad del nivel de gris, textura, etcétera). Asimismo, algunos parámetros que se consideran para ser características globales también se pueden aplicar de forma local, y viceversa (Impedovo y Pirlo, 2008).

Los diferentes descriptores de atributos que son posibles de cuantificar están sintetizados en la tabla 3. Los descriptores comprenden lo siguiente: cálculos y operaciones de ángulos y sus proyecciones, concebidos entre segmentos de rectas y gradientes de variaciones de pixeles, arcos, curvaturas y convexidades de trazos de segmentos de firmas; las operaciones morfológicas usadas en análisis de conectividad, tipos de distancias, la esqueletización, dilataciones, determinación de lazos, variaciones de tonalidades en caso de entropías y texturas, intersecciones o impactos de rayos con pixeles de firmas; las operaciones geométricas con entidades de rectas, masa, centros de gravedad, intersecciones, máximos horizontal y vertical, perímetros, relación altura y ancho y orientaciones. Del mismo modo, se ha identificado el uso de operadores de transformación como DRT y FFT, cuyos coeficientes y parámetros generan elementos de caracterización. También son usados parámetros estadísticos, probabilísticos, desvío estándar, varianza, distribución de pixeles, histogramas, flujo óptico, momento de Hu, entre otros. 
Tabla 3. Descriptores de características usados en la verificación de firmas off-line

\begin{tabular}{|c|c|c|c|}
\hline ID & Descriptor de atributo & Referencias & Cantidad \\
\hline D01 & Angle & Das y Dulger (2009), Pirlo e Impedovo (2013a) & 2 \\
\hline D02 & Arcs & Tselios et al. (2012), Kumar et al. (2012) & 2 \\
\hline D03 & Connectivity & Kovari y Charaf (2013) & 1 \\
\hline D04 & Convexity & Kovari y Charaf (2013) & 1 \\
\hline D05 & Curvature & Bertolini et al. (2010) & 1 \\
\hline D06 & Density & $\begin{array}{l}\text { Batista, Granger y Sabourin (2012), Batista et al. } \\
\text { (2010), Bertolini et al. (2010), Kumar et al. (2012) }\end{array}$ & 4 \\
\hline D07 & Radon Transform (DRT) & Swanepoel y Coetzer (2013) & 1 \\
\hline D08 & Entropy & Kumar et al. (2012) & 1 \\
\hline D09 & Fast Fourier Transformation (FFT) & Wen et al. (2009) & 1 \\
\hline D10 & Gravity center & Das y Dulger (2009), Batista et al. (2010) & 2 \\
\hline D11 & Gradient & Zhang (2010) & 1 \\
\hline D12 & Histogram & Zhang (2010), Pirlo e Impedovo (2013a) & 2 \\
\hline D13 & Hu moment & Radhika et al. $(2010,2011)$ & 2 \\
\hline D14 & Intersections & Pirlo e Impedovo (2013a) & 1 \\
\hline D15 & Mass & Jena et al. (2008), Pirlo e Impedovo (2013a) & 2 \\
\hline D16 & Optical flow & Pirlo e Impedovo (2013b) & 1 \\
\hline D17 & Orientation & Batista et al. (2010), Bertolini et al. (2010) & 2 \\
\hline D18 & Perimeter ring & Wen et al. (2009) & 1 \\
\hline D19 & Pixel distribution & Bertolini et al. (2010) & 1 \\
\hline D20 & Ratio (high/wide) & Das y Dulger (2009) & 1 \\
\hline D21 & Stroke curvature & Batista et al. (2010), Bertolini et al. (2010) & 2 \\
\hline
\end{tabular}

Elaboración propia

En la tabla se observa que uno de los descriptores más utilizados en los trabajos revisados es la densidad.

\subsection{Q3: ¿Qué algoritmo se ha desarrollado para la verificación (clasificación)?}

La clasificación está dividida en tres grupos: template-matching, estadísticos y estructurales; así también, la combinación de métodos de esos grupos dan buenos resultados. En la figura 4 se pueden ver los grupos de clasificadores usados en el reconocimiento de firmas. 
Figura 4. Modelos de clasificadores utilizados en verificación de firmas

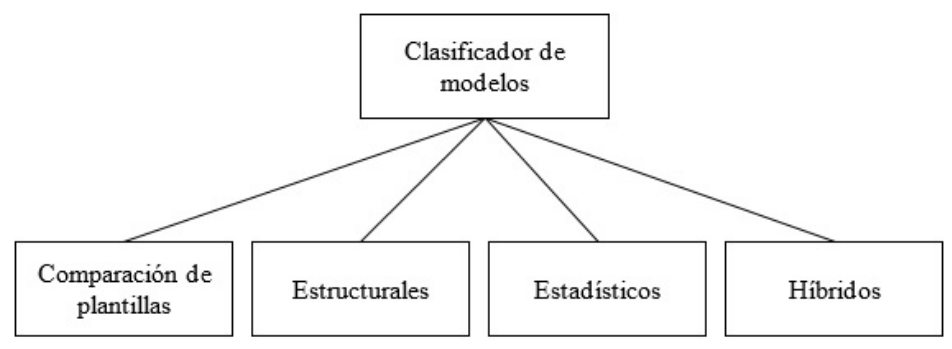

Elaboración propia

\subsubsection{Templates o directos}

Estos clasificadores buscan el grado de similitud entre dos firmas por operaciones directas de sus respectivos vectores de atributos. Por ejemplo, Jena et al. (2008) usa la distancia euclidiana entre cada par de vectores en relación a un valor de tolerancia obtenida por la media de $n$ datos de prueba y sus respectivas varianzas.

Pirlo e Impedovo (2013a) enfocan la clasificación desde la óptica de estabilidad, considerando que firmas genuinas tienen estabilidad menor. Para eso, las imágenes son divididas en regiones de las firmas. Los cosenos de los vectores de las respectivas regiones de dos imágenes de firmas definen un conjunto de valores reales que determinan la variabilidad entre esas imágenes.

\subsubsection{Estructurales}

Modelos basados en árboles de decisión y SVM constituyen el tipo de clasificador estructural. Pirlo e Impedovo (2013b) utilizan una estructura de árbol de decisión alterna (ADT) generadas sobre la base de vectores de atributos de variabilidad de flujo óptico. El ADT contiene nodos de decisión que especifican predicados de condición, y nodos de predicción que contienen números. En la clasificación, cada vector es comparado con los nodos de ADT, y si resulta verdadero, entonces se incrementa en una unidad al contador (voto) de ese vector. Al final, el vector que obtuvo mayor voto se define como variabilidad compatible, validando la firma en análisis.

Los modelos SVM y variantes son bastante usados en verificación de firmas, evidenciados por Swanepoel y Coetzer (2013) y Radhika et al. (2010). Una variante del SVM, conocido como el modelo OC-SVM (one-class support vector machine) es introducido por Guerbai, Chibani y Hadjadji (2015), que consiste en una hiperesfera en la cual al menos un grupo de elementos de entrenamiento está dentro. Guerbai et al. (2015) construyen un modelo one-class SVM (OC-SVM) solo con firmas genuinas, justificando que las instituciones que identifican firmas, como los bancos, solo 
registran firmas genuinas. Los parámetros óptimos del modelo son obtenidos con un conjunto de firmas genuinas registradas. Asumen que cada usuario tiene su propio modelo, pero todos los usuarios tienen los mismos parámetros. Cuando un nuevo usuario es presentado al sistema, el modelo es creado sin calcular sus parámetros; así el sistema es considerado como independiente del escritor.

Zhang (2010) utiliza un SVM multiclase basada en la relación binaria "un-todos", en el que es necesario un SVM para cada clase de los $k$ usados. Considera que el enfoque "un-un" es más intenso computacionalmente, ya que los resultados de cada par de SVM deben ser computados. Zhang compara ese método en relación a otros métodos de clasificación como kNN, regresión logística, Fisher Discriminating Linear (FLD) y MLP. Bertolini et al. (2010) definen un conjunto de clasificadores de decisión parcial basado en SVM, uno para cada clase de firma genuina de un conjunto de personas, alimentados por el grado de disimilaridad definido por la norma de la diferencia de par de vectores de atributos, una genuina y la otra cualquiera (puede ser genuina de la misma persona o de otra considerada como falsa). La decisión final ofrece la fusión de las decisiones parciales por mayoría de votos. En el entrenamiento se consideran casos positivos (combinación de firmas genuinas por persona) y negativos (combinación de firmas de pares de personas diferentes). Se obtienen buenos resultados para cuando el número de divisiones es entre 11 y 15 , obteniéndose error promedio AER entre 6,3 \% y 7,3\%.

Tselios et al. (2012) crean un clasificador basado en SVM por cada firmante con un umbral común para todos. Validan con el dataset GPDS300 y comparan la eficiencia en relación a otros SVM que usan diferentes datasets.

\subsubsection{Estadísticos}

El uso de Artificial Neural Network (ANN) es una alternativa eficiente en reconocimiento de firmas. Kumar et al. (2012) examinan la eficiencia de sus atributos usando dos clasificadores populares: multilayer perceptron y SVM. Radhika et al. (2011) utilizan radial basis function (RBF) alimentados por suma de momentos de MVQC, y alimenta el clasificador forward neural network (FNN).

En Batista et al. (2010), los vectores son clusterizados para generar el codebook como alfabeto de símbolos para entrenar varios HMMs left to right; con diferentes estados demuestran que usar variables codebooks es mejor. Debido a que los vectores de atributos basados en distancias de intersección no son fijos, Kudlacik y Porwik (2014) proponen un modelo difuso de esas distancias para cada persona como un conjunto fuzzy de datos, donde para cada firma nueva a verificar se hace una operación de membresía fuzzy de cada elemento, y luego de todo el conjunto. 
Se aplica un umbral por persona para aceptar o rechazar la firma, ajustando el umbral al peor caso de membresía de la firma de entrenamiento. Para la validación es usado el dataset SVC2004, obteniéndose como resultados para firmas genuinas FAR $0,61 \%$ y FRR $22,16 \%$, y para firmas falsas FAR 1,52 \% y FRR $12,16 \%$.

\subsubsection{Híbridos}

Batista et al. (2012) proponen un modelo híbrido de HMM (como clasificador generativo) y selección dinámica como clasificador discriminativo, un modelo compuesto por clasificadores en dos etapas. Vélez, Sánchez, Moreno y Esteban (2009) usan métricas de coincidencia y distancia, y energía (snake). La primera usa mapa potencial de la imagen, que es 1 si el snake pasa exactamente por medio de los trazos más gruesos de la firma, y baja cuando se desvía de ese trazo. La distancia es la suma de los pixeles en función del ancho del trazo. También usa el enfoque difuso de primer orden de Takagi-Sugeno. La evaluación fue hecha con 6 firmas de cada una de las 56 personas, obteniéndose un EER de 12,5\%.

En la tabla 4 se listan los diversos trabajos agrupados por técnica desarrollada para la clasificación de la firma.

\section{Tabla 4. Métodos de clasificación}

\begin{tabular}{|c|c|c|c|}
\hline Cod & Método de clasificación & Referencias & Cantidad \\
\hline C01 & Artificial Neural Network (ANN) & $\begin{array}{l}\text { Kumar et al. (2012), Randhika et al. (2011), } \\
\text { Das y Dulger (2009), Batista et al. (2010) }\end{array}$ & 4 \\
\hline $\mathrm{CO} 2$ & Decision trees (DT) & Pirlo e Impedovo (2013b) & 1 \\
\hline $\mathrm{CO3}$ & Correlation & $\begin{array}{l}\text { Bhattacharyya, Bandyopadhyay, Das, Ganguly } \\
\text { y Mukherjee (2008) }\end{array}$ & 1 \\
\hline $\mathrm{CO4}$ & Fuzzy Set & Kudlacik y Porwik (2014), Vélez et al. (2009) & 2 \\
\hline $\mathrm{C} 05$ & HMM & Batista et al. (2012), Wen et al. (2009) & 2 \\
\hline $\mathrm{C06}$ & Similarity Distance & $\begin{array}{l}\text { Jena et al. (2008), Vélez et al. (2009), Pirlo e Impedovo } \\
\text { (2013a), Eskander, Sabourin y Granger (2013), } \\
\text { Swanepoel y Coetzer (2013), Rivard, Granger y } \\
\text { Sabourin (2013), Pham, Le y Do (2015), Rico e Iñesta } \\
\text { (2012) }\end{array}$ & 8 \\
\hline $\mathrm{CO7}$ & Stability & Pirlo e Impedovo. (2013a) & 1 \\
\hline $\mathrm{C} 08$ & Statistical & Jena et al. (2008) & 1 \\
\hline C09 & Suport Vector Machine (SVM) & $\begin{array}{l}\text { Swanepoel y Coetzer (2013), Radhika et al. (2010), } \\
\text { Zhang (2010), Bertolini et al. (2010), Tselios et al. } \\
\text { (2012), Guerbai et al. (2015), } \\
\text { Vargas et al. (2011), Kumar et al. (2012), Pal, U., Pal, S. y } \\
\text { Blumenstein (2013), Ferrer et al. (2012) }\end{array}$ & 10 \\
\hline
\end{tabular}


Los métodos de clasificación mayormente usados en los trabajos revisados son basados en SVM, superando largamente a los otros métodos, tal como ilustra la tabla 4.

\subsection{Q4: ¿Qué datasets se han utilizado para validar los métodos de verificación de firmas?}

Las databases usadas en los trabajos analizados son GPDS, referenciados a sus datasets GPDS300, GPDS160 y GPDS960 GraySignatures. Otras databases regularmente utilizadas en verificación de firmas son CEDAR, MUKOS, SVC2004, SigComp2011, MCYT100, Brazilian database; Hindi database; GAVAB, Greek database, Dolfing dataset, Tobacco-800 y databases privados, tal como se muestra en la tabla 5 . Asimismo, en la tabla 6 se resumen las características que constituyen las databases conocidas.

Tabla 5. Base de datos de firmas usadas

\begin{tabular}{|l|l|c|}
\hline \multicolumn{1}{|c|}{ Base de datos } & \multicolumn{1}{c|}{ Referencias } & 8 \\
\hline GPDS300 & $\begin{array}{l}\text { Kumar et al. (2012); Telios et al. (2012), Pirlo e Impedovo (2013a), Pirlo } \\
\text { e Impedovo. (2013b), Zhang (2010); Guerbai et al. (2015); Eskander et } \\
\text { al. (2013), Tselios et al. (2012) }\end{array}$ & 2 \\
\hline CEDAR & Guerbai et al. (2015); Kumar et al. (2012) & 5 \\
\hline MCYT100 & $\begin{array}{l}\text { Wen et al. (2009), Rico e Iñesta (2012); Radhika et al. (2010); } \\
\text { Radhika et al. (2011); Ferrer } \text { et al. (2012) }\end{array}$ & 1 \\
\hline SVC2004 & Kudlacik y Porwik (2014) & 1 \\
\hline SigComp2011 & Pham et al. (2015) & 1 \\
\hline Hidu Sig. Database & Pal et al. (2013) & 3 \\
\hline Brazilian Database & Batista et al. (2010, 2012); Eskander et al. (2013) & 1 \\
\hline Dolfing data set & Swanepoel y Coetzer (2013) & 5 \\
\hline Private Database & $\begin{array}{l}\text { Vélez et al. (2009), Tselios et al. (2012), Rivard et al. (2013), Jena } \\
\text { et al. (2008); Bertolini et al. (2010) }\end{array}$ \\
\hline
\end{tabular}

Tabla 6. Bases de datos y sus atributos

\begin{tabular}{|l|c|c|c|c|}
\hline \multicolumn{1}{|c}{ Conjunto de datos } & $\begin{array}{c}\text { Cantidad de } \\
\text { firmantes }\end{array}$ & $\begin{array}{c}\text { Cantidad de firmas } \\
\text { genuinas }\end{array}$ & $\begin{array}{c}\text { Cantidad de } \\
\text { falsificaciones }\end{array}$ & $\begin{array}{c}\text { Total de } \\
\text { firmas }\end{array}$ \\
\hline $\begin{array}{l}\text { GPDS (Univ. Las Palmas } \\
\text { de Gran Canaria) }\end{array}$ & 300 & 24 & 30 & 16200 \\
\hline GPDS-160 & 160 & 24 & 30 & 8640 \\
\hline GPDS960 GraySignature & 75 & 24 & 30 & 4125 \\
\hline CEDAR (SUNY, Buffalo) & 55 & 24 & 24 & 2640 \\
\hline Brazilian & 168 & 80 & 20 & 7920 \\
\hline MCYT-100 & 75 & 15 & 15 & 2250 \\
\hline SigComp2011 & 64 & 773 & 1071 & 1844 \\
\hline Hindi Database & 100 & 24 & 30 & 5400 \\
\hline SVC2004 & 40 & 20 & 20 & 1600 \\
\hline Dolfing data set & 51 & 30 & 60 & 4530 \\
\hline
\end{tabular}

Elaboración propia 
En la tabla 5 se observa que un mayor número de trabajos usan GPDS300, seguidos por trabajos que utilizan MCYT100 y databases privados, mientras que el dataset Brazilian es el tercer preferido por los trabajos. La preferencia por datasets privados posiblemente justifique que no todos están disponibles en forma gratuita o son de simple acceso.

\subsection{Q5: ¿Cuál es el rendimiento que presentan los trabajos de verificación de firmas?}

Las diferentes databases utilizadas en los trabajos para validar sus métodos propuestos son resumidas en la tabla 7, en función de las métricas AER y EER. A los trabajos que solo muestran FAR y FRR se le calculó el AER, con el objetivo de uniformizar los valores de medición.

Tabla 7. Rendimiento y métricas usadas en los trabajos revisados

\begin{tabular}{|c|c|c|c|c|c|}
\hline Referencias & Base de datos & FAR & FRR & AER & EER \\
\hline Zhang et al. (2010) & GPDS300 & 3,87 & 3,98 & 3,93 & \\
\hline Guerbai et al. (2015) & & & & 15,95 & \\
\hline Eskander et al. (2013) & & & & 12,50 & \\
\hline Kumar et al. (2012) & & 8,33 & 13,76 & 11,05 & \\
\hline Pirlo e Impedovo (2013a) & & & & & 9,60 \\
\hline Pirlo e Impedovo (2013b) & & & & & 5,40 \\
\hline Tselios et al. (2012) & & & & & 2,00 \\
\hline Guerbai et al. (2015) & CEDAR & & & 7,83 & \\
\hline Kumar et al. (2012) & & 8,33 & 12,76 & 10,55 & \\
\hline Vélez et al. (2009) & Privates & & & & 12,50 \\
\hline Tselios et al. (2012) & & & & & 2,00 \\
\hline Ferrer et al. (2012) & MCYT100 & & & & 17,00 \\
\hline Radhika et al. (2010) & & 10,00 & 8,00 & 9,00 & \\
\hline Radhika et al. (2011) & & 10,0 & 7,00 & 8,50 & \\
\hline Kudlacik y Porwik (2014) & SVC2004 & 1,52 & 12,16 & 6,84 & \\
\hline Pham et al. (2015) & SigComp2011 & 12,05 & 12,35 & 12,20 & \\
\hline Eskander et al. (2013) & Brazilian & & & 7,34 & \\
\hline Batista et al. $(2010,2012)$ & & & & 7,79 & \\
\hline Swanepoel y Coetzer (2013) & Dolfing & & & & 3,78 \\
\hline Pal et al. (2013) & Hindi & 7,12 & 11,50 & 9,31 & \\
\hline
\end{tabular}

Elaboración propia

Las mejores métricas de AER y EER se obtuvieron usando GPDS300, logrando alcanzar un AER mínimo de 3,93 por Zhang et al (2010) y un EER mínimo de 2,00 por Telios et al. (2012). 


\section{Conclusiones}

i. En este artículo se han revisado los trabajos de verificación automática de firmas, así como los algoritmos y técnicas que ayudan al proceso de verificación.

ii. Gran parte de los trabajos presentados extraen características comunes, pero ninguno usa el método de extracción de características realizado por un especialista grafotécnico.

iii. Debido al amplio uso de la firma como medio de identificación personal, en los últimos años se están presentando trabajos de verificación automática de firmas con mejoras en los niveles de error y precisión cada vez más óptimas.

\section{Referencias}

Batista, L., Granger, E., y Sabourin, R. (2010). Improving performance of HMM-based off-line signature verification systems through a multi-hypothesis approach. International Journal on Document Analysis and Recognition (IJDAR), 13(1), 33-47. DOI: 10.1007/s10032-009-0101-0

Batista, L., Granger, E., y Sabourin, R. (2012). Dynamic selection of generative-discriminative ensembles for off-line signature verification. Pattern Recognition, 45(4), 1326-1340. DOI: 10.1016/j.patcog.2011.10.011

Bertolini, D., Oliveira, L., Justino, E., y Sabourin, R. (2010). Reducing forgeries in writer-independent off-line signature verification through ensemble of classifiers. Pattern Recognition, 43(1), 387-396. DOI: 10.1016/j.patcog.2009.05.009

Bhattacharyya, D., Bandyopadhyay, S., Das, P., Ganguly, D., y Mukherjee, S. (2008). Statistical approach for offline handwritten signature verification. Journal of Computer Science, 4(3), 181-185. DOI: 10.3844/jcssp.2008.181.185

Das, M. y Dulger, L. (2009). Signature verification (SV) toolbox: Application of PSO-NN. Engineering Applications of Artificial Intelligence, 22(4-5), 688-694. DOI: 10.1016/j.engappai.2009.02.005

Eskander, G., Sabourin, R., y Granger, E. (2013). Hybrid writer-independent-writer-dependent offline signature verification system. IET Biometrics, 2(4), 169-181. DOI: 10.1049/ iet-bmt.2013.0024

Ferrer, M., Vargas, J., Morales, A., y Ordóñez, A. (2012). Robustness of Offline Signature Verification Based on Gray Level Features. IEEE Transactions on Information Forensics and Security, 7(3), 966-977. DOI: 10.1109/TIFS.2012.2190281

Guerbai, Y., Chibani, Y., y Hadjadji, B. (2015). The effective use of the one-class SVM classifier for handwritten signature verification based on writer-independent parameters. Pattern Recognition, 48(1), 103-113. DOI: 10.1016/j.patcog.2014.07.016 
Impedovo, D., y Pirlo, G. (2008). Automatic Signature Verification: The State of the Art. IEEE Transactions on Systems, Man, and Cybernetics, Part C (Applications and Reviews), 38(5), 609-635. DOI: 10.1109/TSMCC.2008.923866

Jena, D., Majhi, B., Panigrahy, S., y Jena, S. (2008). Improved offline signature verification scheme using feature point extraction method. En 2008 7th IEEE International Conference on Cognitive Informatics, 475-480. DOI: 10.1109/COGINF.2008.4639204

Kitchenham, B., Brereton, O., Budgen, D., Turner, M., Bailey, J., y Linkman, S. (2009). Systematic literature reviews in software engineering - A systematic literature review. Information and Software Technology, 51(1), 7-15. DOI: 10.1016/j.infsof.2008.09.009

Kovari, B., y Charaf, H. (2013). A study on the consistency and significance of local features in off-line signature verification. Pattern Recognition Letters, 34(3), 247-255. DOI: 10.1016/j. patrec.2012.10.011

Kudłacik, P., y Porwik, P. (2014). A new approach to signature recognition using the fuzzy method. Pattern Analysis and Applications, 17(3), 451-463. DOI: 10.1007/s10044-012-0283-9

Kumar, R., Sharma, J., y Chanda, B. (2012). Writer-independent off-line signature verification using surroundedness feature. Pattern Recognition Letters, 33(3), 301-308. DOI: 10.1016/j. patrec.2011.10.009

Leclerc, F., y Plamondon, R. (1994). Automatic signature verification: the state of the art-19891993. International Journal of Pattern Recognition and Artificial Intelligence, 8(3), 643-660. DOI: $10.1142 / S 0218001494000346$

Pal, U., Pal, S., y Blumenstein, M. (2013). Off-line verification technique for Hindi signatures. IET Biometrics, 2(4), 182-190. DOI: 10.1049/iet-bmt.2013.0016

Pham, T., Le, H., y Do, N. (2015). Offline handwritten signature verification using local and global features. Annals of Mathematics and Artificial Intelligence, 75(1-2), 231-247. DOI: 10.1007/ s10472-014-9427-5

Pirlo, G., e Impedovo, D. (2013a). Cosine similarity for analysis and verification of static signatures. IET Biometrics, 2(4), 151-158. DOI: 10.1049/iet-bmt.2013.0012

Pirlo, G., e Impedovo, D. (2013b). Verification of Static signatures by optical flow analysis. IEEE Transactions on Human-Machine Systems, 43(5), 499-505. DOI: 10.1109/THMS.2013.2279008

Plamondon, R., y Lorette, G. (1989). Automatic signature verification and writer identification the state of the art. Pattern Recognition, 22(2), 107-131. DOI: 10.1016/0031-3203(89)90059-9

Radhika, K., Venkatesha, M., y Sekhar, G. (2010). Off-Line signature authentication based on moment invariants using support vector machine. Journal of Computer Science, 6(3), 305-311. DOI: 10.3844/jcssp.2010.305.311 
Radhika, K., Venkatesha, M., y Sekhar, G. (2011). Signature authentication based on subpattern analysis. Applied Soft Computing, 11(3), 3218-3228. DOI: 10.1016/j.asoc.2010.12.024

Rico, J., e Iñesta, J. (2012). Confidence voting method ensemble applied to off-line signature verification. Pattern Analysis and Applications, 15(2), 113-120. http://doi.org/10.1007/ s10044-012-0270-1

Rivard, D., Granger, E., y Sabourin, R. (2013). Multi-feature extraction and selection in writer-independent off-line signature verification. International Journal on Document Analysis and Recognition (IJDAR), 16(1), 83-103. DOI: 10.1007/s10032-011-0180-6

Shekar, B., Bharathi, R., Kittler, J., Vizilter, Y., y Mestestskiy, L. (2015). Grid structured morphological pattern spectrum for off-line signature verification. En 2015 International Conference on Biometrics (ICB) (430-435). IEEE. DOI: 10.1109/ICB.2015.7139106

Swanepoel, J., y Coetzer, J. (2013). A robust dissimilarity representation for writer-independent signature modelling. IET Biometrics, 2(4), 159---168. DOI: 10.1049/iet-bmt.2013.0011

Tselios, K., Zois, E., Siores, E., Nassiopoulos, A., y Economou, G. (2012). Grid-based feature distributions for off-line signature verification. IET Biometrics, 1(1), 72-81. DOI: 10.1049/ iet-bmt.2011.0011

Vargas, J., Ferrer, M., Travieso, C., y Alonso, J. (2011). Off-line signature verification based on grey level information using texture features. Pattern Recognition, 44(2), 375-385. DOI: 10.1016/j. patcog.2010.07.028

Vélez, J., Sánchez, Á., Moreno, B., y Esteban, J. (2009). Fuzzy shape-memory snakes for the automatic off-line signature verification problem. Fuzzy Sets and Systems, 160(2), 182-197. DOI: 10.1016/j.fss.2008.05.021

Wen, J., Fang, B., Tang, Y., y Zhang, T. (2009). Model-based signature verification with rotation invariant features. Pattern Recognition, 42(7), 1458-1466. DOI: 10.1016/j.patcog.2008.10.006

Zhang, B. (2010). Off-line signature verification and identification by pyramid histogram of oriented gradients. International Journal of Intelligent Computing and Cybernetics, 3(4), 611-630. DOI: 10.1108/17563781011094197 